\title{
共价有机框架在传感中的研究进展
}

\author{
于 歌 汪 成* \\ (武汉大学化学与分子科学学院 武汉 430072)
}

\begin{abstract}
摘要 共价有机框架(Covalent Organic Frameworks, COFs)作为一种由有机结构单元通过共价键连接而成的晶态有机多 孔材料, 具有结晶性好、密度低、比表面积高以及结构可设计性强等特点, 已在分子吸附与分离、催化、光电及能源 等领域展现出巨大的应用潜力. 近年来, COFs 因其固有的结构特点，在传感领域也逐渐引起了科研工作者的广泛关注. 主要综述了 COFs 在爆炸物传感、湿度传感、金属离子传感、 $\mathrm{pH}$ 传感、生物传感、气体传感等领域中的研究进展, 并 对其发展前景进行了展望.
\end{abstract}

关键词 共价有机框架; 传感; 爆炸物; 金属离子; 气体分子

\section{Research Progress of Covalent Organic Frameworks in Sensing}

\author{
$\mathrm{Yu}, \mathrm{Ge} \quad$ Wang, Cheng* \\ (College of Chemistry and Molecular Sciences, Wuhan University, Wuhan 430072)
}

\begin{abstract}
Covalent organic frameworks (COFs) are an emerging class of porous crystalline organic materials connected by covalent bonds. Owing to their high crystallinity, low density, large surface area and designable structures, COFs have potential applications in molecular adsorption and separation, catalysis, optoelectronic devices, and energy storage. Recently, due to their inherent characteristics, COFs have attracted a lot of interests in sensing. The research progress of COFs in sensing, including explosive sensing, humidity sensing, metal ions sensing, $\mathrm{pH}$ sensing, biosensing and gas sensing is summarized. Finally, a perspective of the application of COFs in sensing is given.
\end{abstract}

Keywords covalent organic frameworks; sensing; explosive; metal ion; gas molecule

共价有机框架(Covalent Organic Frameworks, $\mathrm{COFs}$ )是一类由有机结构单元通过共价键连接而成的晶 态有机多孔材料 ${ }^{[1]}$. 相较于共轭微孔聚合物(CMP) $)^{[2]}$ 、多 孔芳香骨架 $(\mathrm{PAF})^{[3]}$ 、超交联聚合物 $(\mathrm{HCP})^{[4]}$ 等无定形有 $^{-}$ 机多孔材料而言, $\mathrm{COFs}$ 具有以下特点: 首先, $\mathrm{COFs}$ 具有 良好的结晶性, 其结构可通过 X 射线衍射及电子衍射等 表征手段进行有效地解析, 有利于研究者对 COFs 构效 关系的理解. 其次, COFs 具有极强的可设计性, 研究者 除可通过丰富的有机合成手段对其有机组分进行设计、 合成, 还可通过框架化学对 COFs 骨架进行设计, 进而 实现 $\mathrm{COFs}$ 的定向构筑与功能化. 此外, COFs 作为一种 由轻元素(如 C, H, N 等)组成的高孔隙率骨架结构, 具有 较低的密度(低至 $0.13 \mathrm{~g} \cdot \mathrm{cm}^{-3}$ )和较高的比表面积(可达 $\left.5083 \mathrm{~cm}^{2} \cdot \mathrm{g}^{-1}\right)^{[5]}$. 因此, 自 2005 年 Yaghi 等 ${ }^{[6]}$ 报道首例 $\mathrm{COF}$ 以来, $\mathrm{COFs}$ 已在分子吸附与分离 ${ }^{[7]}$ 、催化 ${ }^{[8]}$ 、光

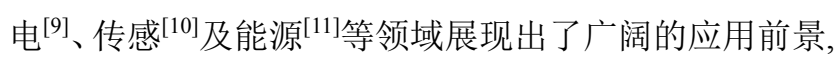
取得了重要的研究进展.

近年来, $\mathrm{COFs}$ 作为一种稳定性高、可设计性强的晶 态有机多孔材料, 在传感领域表现出很多独特的优势: (1)利用 COFs 可设计性强的特点, 研究者可将特定功能 基元引入到其骨架中, 实现目标分析物的选择性检测; (2) COFs 规整开放的孔道结构有利于目标分析物同作 用位点接触，实现传感过程; (3)由共价键连接而成的 $\mathrm{COFs}$ 具有良好的稳定性，可在传感过程中保持结构的 完整, 实现材料的重复利用. 基于上述优势, COFs 材料 在传感领域中的应用已取得了一系列进展 ${ }^{[10]}$.

目前, $\mathrm{COFs}$ 领域的综述主要关注了 $\mathrm{COFs}$ 的结构设 计与合成以及其综合性应用 ${ }^{[1,12]}$. 相比之下, 单独关注 某一领域(如能源存储 ${ }^{[11 \mathrm{~d}, 11 \mathrm{e}]}$ 、催化 ${ }^{[8]}$ 以及传感 $\left.{ }^{[10 \mathrm{~d}]}\right)$ 的综 述较少. 为此, 本综述对 COFs 在传感领域中的应用研

\footnotetext{
* Corresponding author. E-mail: chengwang@whu.edu.cn

Received March 7, 2020; revised April 5, 2020; published online April 17, 2020.

Project supported by the National Natural Science Foundation of China (Nos. 21772149, 21975188).

国家自然科学基金(Nos. 21772149, 21975188)资助项目.
} 
究进行了文献综述, 并对其未来趋势和主要挑战进行总 结和展望, 以期为 COFs 在传感领域的发展提供新思路.

\section{COFs 在传感中的应用}

传感是根据探针与目标分析物接触所产生的物理 化学性质的变化实现分析对象的检测, 在食品与社会安 全的控制及疾病的检测中具有重要的意义. 一般而言, 当检测物同 COFs 连接键或功能基元发生作用时, COFs 本身颜色及光电性质发生一定改变, 根据这些性质的改 变, 即可实现目标分析物的检测 ${ }^{[13]}$. 本部分根据 COFs 传感对象进行分类, 分别对 COFs 在爆炸物传感、湿度 传感、金属离子传感、 $\mathrm{pH}$ 传感、生物传感、气体传感 等应用中的研究进展进行总结.

\subsection{COFs 在爆炸物传感中的应用}

爆炸物在人类对生活环境的改造过程中起到了无 可比拟的作用, 但同时也给社会的安定带来了重大的威 胁. 因此, 发展高效、快速和灵敏的爆炸物检测方法对 恐怖分子的控制及社会安全的保障具有重要的意义. COFs 因其结构和功能的可设计性在爆炸物传感领域中 已展现出了重大潜力. 本部分将对 COFs 在爆炸物传感 方面的应用进行总结与概括.

苂光传感具有响应快速和检测灵敏等特点, 是一种 爆炸物检测的重要方法. 引入发光基团, 构筑具有良好
发光性能的 COFs，是 COFs 在传感应用中的常用方 法 $^{[14]}$. 2013 年, Jiang 等 ${ }^{[14 \mathrm{~d}]}$ 将具有良好发光性能的 1,3,6,8-四(4-甲醛基苯基)萠作为前体，在溶剂热条件下 构筑了具有良好发光性能的二维 COF (Py-Azine, 图 1a). 研究发现, 当 Py-Azine 乙腈悬浮液中加入 $70 \mathrm{mg} ・$ $\mathrm{L}^{-1}$ 检测物时, Py-Azine 的苂光可被 2,4,6-三硝基苯酚 (TNP)有效猝灭 $(69 \%$, 图 1b). 而当检测物为 2,4-二硝基 苯酚(DNP)、2,4-二硝基甲苯(DNT)、2-硝基苯酚(NP)以 及 2-硝基甲苯(NT)时, Py-Azine 的苂光仅能分别猝灭 $13 \%, 5 \%, 3 \%$ 及 3\%(图 1c). 上述结果表明 Py-Azine 可用 于爆炸物 TNP 的选择性传感. Jiang 等认为检测物分子 中的酚羟基与 Py-Azine 中氮原子形成氢键，使 COFs 的 $\pi$ 电子密度降低，苂光被猝灭; 而 TNP 的三个硝基进一 步降低了 COFs 的 $\pi$ 电子密度, 使 Py-Azine 对其表现出 较高的响应性与选择性.

尽管荧光二维 COFs 表现出了良好的传感特性, 其 层与层之间的 $\pi-\pi$ 堆积却在一定程度上限制了其荧光性 能以及 COFs 与客体分子间的相互作用 ${ }^{[14 a]}$. 为此, Banerjee 等 ${ }^{[10 a]}$ 通过超声剥离的方法, 将二维 COF (TfpBDH) 进行液相剥离, 制备了基于亚胺键的二维共价有机纳米 片(TfpBDH-CONs). 苂光测试发现, TfpBDH-CONs 苂光 性能较 $\mathrm{TfpBDH}$ 大幅提升。进一步实验结果表明, TfpBDH-CONs 对 TNP 可分别展现出独特的荧光猝灭增 (a)

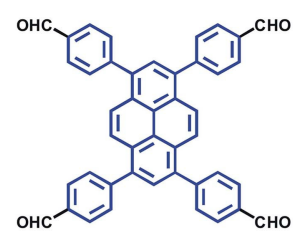

(b)

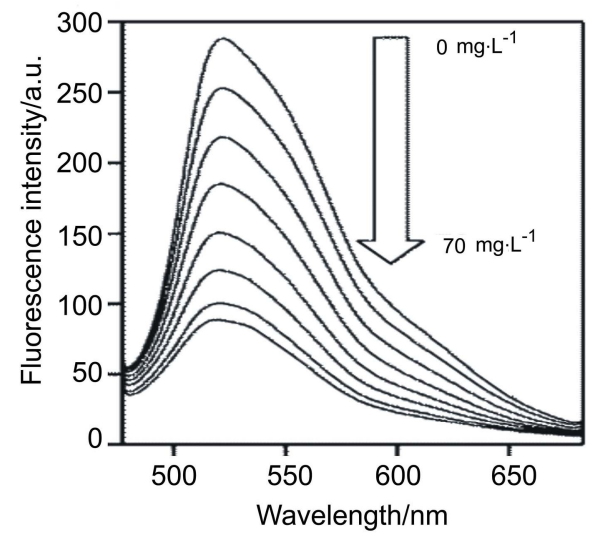

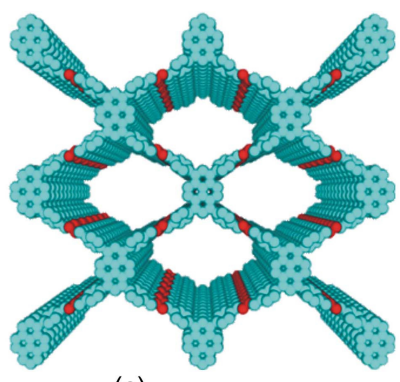

(c)

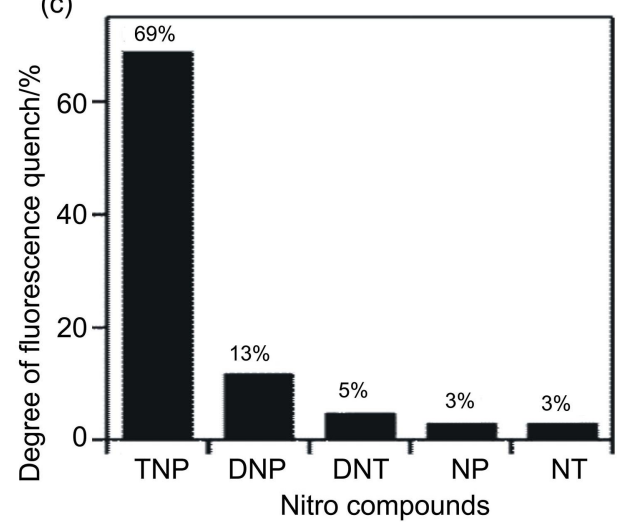

图 1 (a) Py-Azine COF 的合成及结构示意图、(b) Py-Azine COF 在不同浓度 TNP 下 $\left(0 \sim 70 \mathrm{mg} \cdot \mathrm{L}^{-1}\right)$ 的荧光变化和(c) Py-Azine COF 对不同硝基化合物的选择性 $\left(70 \mathrm{mg} \cdot \mathrm{L}^{-1}\right)$

Figure 1 (a) Synthesis and structure of Py-Azine COF, (b) fluorescence quenching of the Py-Azine COF upon addition of TNP $(0 \sim 70$ $\left.\mathrm{mg} \cdot \mathrm{L}^{-1}\right)$, and (c) degree of fluorescence quenching upon addition of the nitro compounds $\left(70 \mathrm{mg} \cdot \mathrm{L}^{-1}\right)$ 
强传感性能. 当 TfpBDH-CONs 处于分散状态时, TNP 可与其形成主客体氢键, 猝灭其荧光. 而当 $\mathrm{TfpBDH}-$ CONs 处于聚集态时, TNP 可通过对其亚胺键中氮原子 的质子化作用使其荧光增强. 此外, 在胺蒸气(TEA)作 用下与 TNP 作用后的 TfpBDH-CONs 黄色试纸会在几秒 内恢复到其初始灰色, 实现可逆检测.

此外, 将荧光基团引入三维 COFs 也可构筑具有较 好苂光性能的 COFs, 进而用于爆炸物传感. 2016 年, 汪 成等 ${ }^{[14 \mathrm{e}]}$ 利用四面体和平面四边形的前体, 通过 $[4+4]$ 缩 合反应成功合成了第一例三维荧光 COF (3D-Py-COF) (图 2a, 2b). 研究发现, 3D-Py-COF 具有较强的黄绿色荧 光, 在 $N, N$-二甲基甲酰胺(DMF) 悬浮液中最大发射波长 为 $484 \mathrm{~nm}\left(\lambda_{\mathrm{ex}}=408 \mathrm{~nm}\right)$. 此外, 苦味酸(PA)对 3D-PyCOF 的荧光具有明显的猝灭效果, 且随着苦味酸浓度 的增加荧光猝灭效果逐渐增加; 当 PA 浓度增加至 20 $\mathrm{mg} \cdot \mathrm{L}^{-1}$ 时, $75 \%$ 以上 $\mathrm{COF}$ 苂光被猝灭, 猝灭常数为 $3.1 \times$

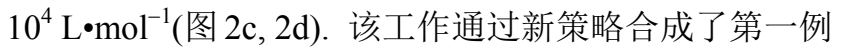
荧光三维 COF, 对三维 COFs 的发展具有重要意义. 随 后, 他们构筑了三维四苯乙烯 COF (3D-TPE-COF), 同 样也可以用于 PA 的检测 ${ }^{[9 e]}$.

\section{$1.2 \mathrm{COFs}$ 在湿度传感中的应用}

环境湿度的检测和控制对人类的生产和生存具有 重要的意义. 而传统的湿度检测方法(如卡尔-费休滴定

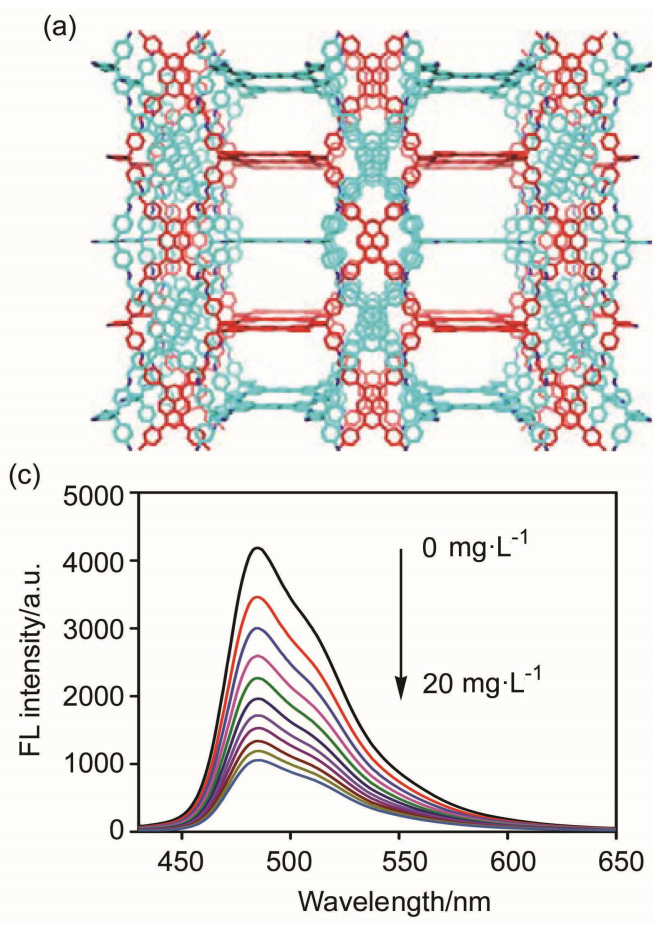

法和色谱法等)操作繁琐、分析时间长，不利于湿度的快 捷检测 ${ }^{[15]}$. COFs 在传感中具有读取方便、响应快速以及 可重复使用等诸多优势，已在该领域展现出了巨大潜 力 $^{[16]}$. 本部分将对 COFs 在湿度传感方面的应用进行讨 论.

COFs 在不同湿度下展现出的性质(如颜色)改变, 可用于湿度的检测. 2013 年, 刘习奎等 ${ }^{[16 c]}$ 以 2,6 -二醛 基-1,5-二羟基䒬(DHNDA)与 2,4,6-三(4-氨基苯基)吡啶 (TAPP)为前体合成了 $\mathrm{COF}$ 微球, 并通过溶解-重结晶的 方法实现了 $\mathrm{COF}$ 微球到纳米纤维的转变, 随后将其生 长到聚酰胺微纤维的表面, 制备了具有比色湿度响应的 功能化 COF 纳米纤维/芳纶织物(图 3a). 测试发现, 该复 合物在干燥气氛 [相对湿度 $(\mathrm{RH})$ 为 $20 \%$ ] 中显示橙黄色, 而随着相对湿度的增加 $(20 \% \sim 100 \%)$, COFs 骨架发生一 定程度醇酮异构, 复合物颜色逐渐从橙黄色变为橙红 色，可用于环境湿度的肉眼可见检测(图 3b).

此外，通过 COFs 阻抗性能的变化也可实现环境湿 度的检测. 2017 年, $\mathrm{Pal}$ 等 ${ }^{[16 d]}$ 通过硼酸酯缩合反应合成 了基于 Truxene 的 COF-TXDBA, 湿度传感结果表明, COF-TXDBA 所处环境相对湿度从 11\%增加到 $98 \%$ 时, 其阻抗产生 3 个数量级的变化, 响应时间和恢复时间分 别为 37 和 $42 \mathrm{~s} . \mathrm{Pal}$ 等认为, 当空气中湿度较低时, 水分 子与 COF-TXDBA 中的硼酸酯连接键发生相互作用,

(b)

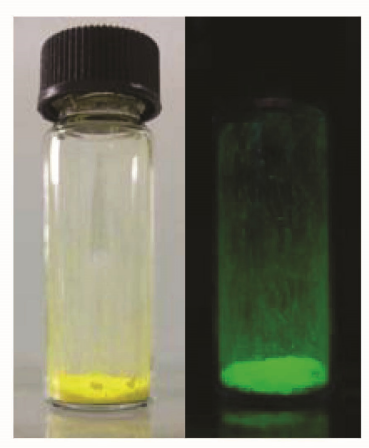

(d)

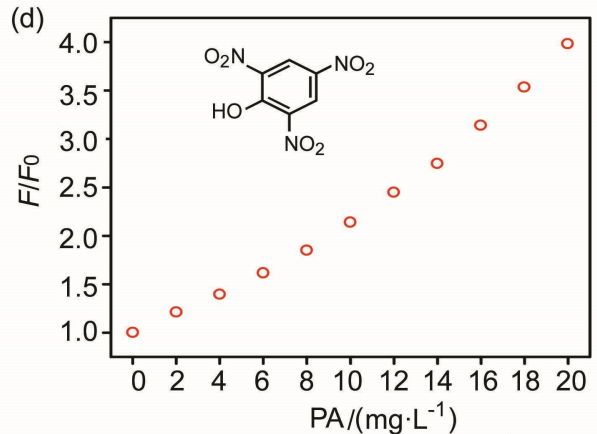

图 2 (a) 3D-Py-COF 结构示意图、(b)可见光(左)与紫外光(右)下 3D-Py-COF 粉末照片和(c, d) 3D-Py-COF 苂光在 DMF 溶液中加 入苦味酸 $\left(\mathrm{PA},\left(0 \sim 20 \mathrm{mg} \cdot \mathrm{L}^{-1}\right)\right.$ 后的猝灭情况

Figure 2 (a) Structure of 3D-Py-COF, (b) photography of the 3D-Py-COF powders under visible light (left) and UV light (right), and (c, d) fluorescence quenching upon addition of PA $\left(0 \sim 20 \mathrm{mg} \bullet \mathrm{L}^{-1}\right)$ in DMF 

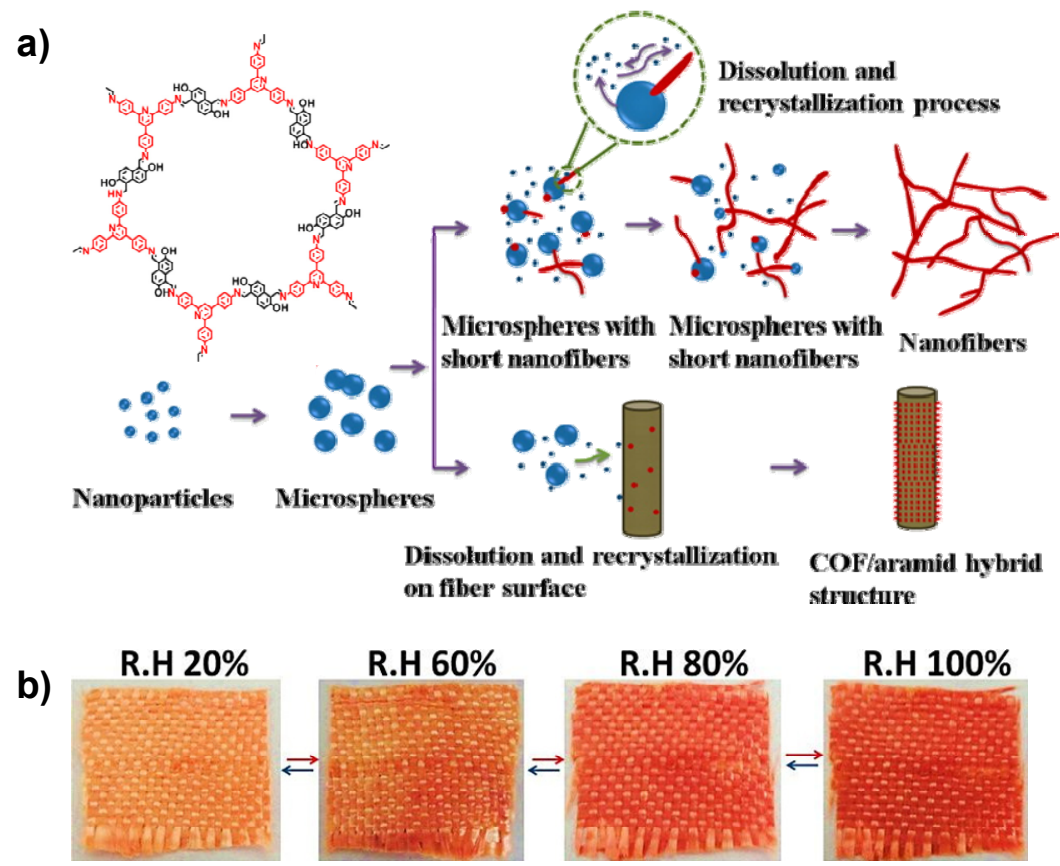

图 3 (a) COF 纳米纤维/芳纶织物的制备和(b)不同相对湿度下的可逆响应性颜色变化

Figure 3 (a) Construction of COF/Aramid fabrics, and (b) digital photographs of the reversible humidity-responsive color-changes of $\mathrm{COF} /$ aramid fabrics under different relative humidity

吸附于 $\mathrm{COF}$ 表面形成间断的水分子层, 水分子运动受 阻, 阻抗较高; 当湿度升高时, 水分子会在 COF 孔道中 聚集形成质子传输通道, 从而使其阻抗大大降低.

除了对空气中湿度的检测, COFs 还可用于有机溶 剂中水份含量的检测. 2017 年, 严秀平等 ${ }^{[16 e]}$ 选用富含羟 基的 2,5-二羟基对苯二甲醛与 4,4',4"-(1,3,5-三嗪-2,4,6三基)三苯胺为前体构筑了 COF-TzDa, 该 COF 中所存 在羟基可与亚胺键中的氮原子形成分子内氢键. 当 COF-TzDa 与水分子接触时, 水分子与羟基产生竞争效 应, 破坏分子内氢键, 使 COF-TzDa 的荧光性能发生改 变, 从而实现水分子的传感. 值得一提的是, 该 $\mathrm{COF}$ 因 分子内电荷转移(ICT) 和激发态分子内质子转移(ESIPT) 效应具有两个主要的苂光发射波长, 均对水分子表现出 灵敏的苂光响应行为, 可用于有机溶剂中水分含量的比 率型荧光检测. 有机溶剂中水分含量的增加, 不仅可破 坏羟基与氮原子之间的分子内氢键, 使得 COF $590 \mathrm{~nm}$ 处荧光增强; 还可破坏原有 ICT 过程, 使得 COF $500 \mathrm{~nm}$ 处苂光减弱. 此外, 严秀平等发现所检测结果与气相色 谱法结果无明显差异, 说明 COF-TzDa 可用于有机溶剂 中水分含量的准确检测, 具有潜在的应用前景.

\section{$1.3 \mathrm{COFs}$ 在重金属离子传感中的应用}

重金属具有较高的毒性且极易在生物体内富集，使 用过程中的痕量释放就会导致多种严重疾病. 因此, 重 金属离子的痕量检测对环境的监测和食品安全的控制 至关重要 ${ }^{[17]}$. 根据 $\mathrm{COFs}$ 的可设计性, 可将与重金属离
子具有相互作用的功能基团引入到 COFs 孔道中, 进而 实现重金属离子的传感 ${ }^{[18]}$. 此外, COFs 在水相中对重金 属离子进行检测的过程展现出优越的稳定性, 并可循环 使用.

金属录在生物医疗与日常生活中均有着广泛的应 用，但其泄露和污染对公众环境产生了巨大威胁，其痕 量监测和去除具有重要意义. 为此, 可将与 $\mathrm{Hg}^{2+}$ 强相互 作用的硫醇(醚)引入到 $\mathrm{COFs}$ 孔道中, 构筑对 $\mathrm{Hg}^{2+}$ 具有 很好识别性能的 COFs 材料 ${ }^{[10 b, 18 a] .2016}$ 年, 王为等 ${ }^{[10 b]}$ 通过酰腙键构筑了孔道内修饰有硫醚功能基团的荧光 COF-LZU8，将其用于 $\mathrm{Hg}^{2+}$ 吸附测试发现，COF-LZU8 与 $\mathrm{Hg}^{2+}$ 作用之后苂光被猝灭, 且在浓度为 $6 \mathrm{mg} / \mathrm{L}$ 的 $\mathrm{Hg}^{2+}$ 溶液中荧光猝灭效率高达 $83 \%$. 此外, COF-LZU8 对 $\mathrm{Hg}^{2+}$ 的吸附量可达 $236 \mathrm{mg} \cdot \mathrm{g}^{-1}$, 表明 COF- LZU8 实 现 $\mathrm{Hg}^{2+}$ 传感的同时进行有效去除(图 4a, 4b). 值得一提 的是, COF-LZU8 具有良好的稳定性, 可用于多种复杂 水质中 $\mathrm{Hg}^{2+}$ 的吸附, 并在吸附/脱附 3 次循环后保持框 架的完整，具备一定的应用前景(图 4c).

近日, 邱建丁等 ${ }^{[18 \mathrm{a}]}$ 构筑了基于碳酰肼链接键的二 维荧光 TFPPy-CHYD, 并将其用于 $\mathrm{Hg}^{2+}$ 的高效选择性 检测，检测限可达 $3 \mathrm{~g} / \mathrm{L}$.

此外, $\mathrm{COFs}$ 还可用于 $\mathrm{Cu}^{2+[19]}$ 等金属离子的检测。 如 2016 年, 刘晓明等 ${ }^{[19]}$ 以 1,3,5-三(3'-叔丁基-4'-羟基-5'苯甲醛基)苯和水合肼为前体构筑了用于 $\mathrm{Cu}^{2+}$ 传感的苂 光 COF-JLU3(图 5a 5c). 研究结果表明, COF-JLU3 对 


\section{a)}

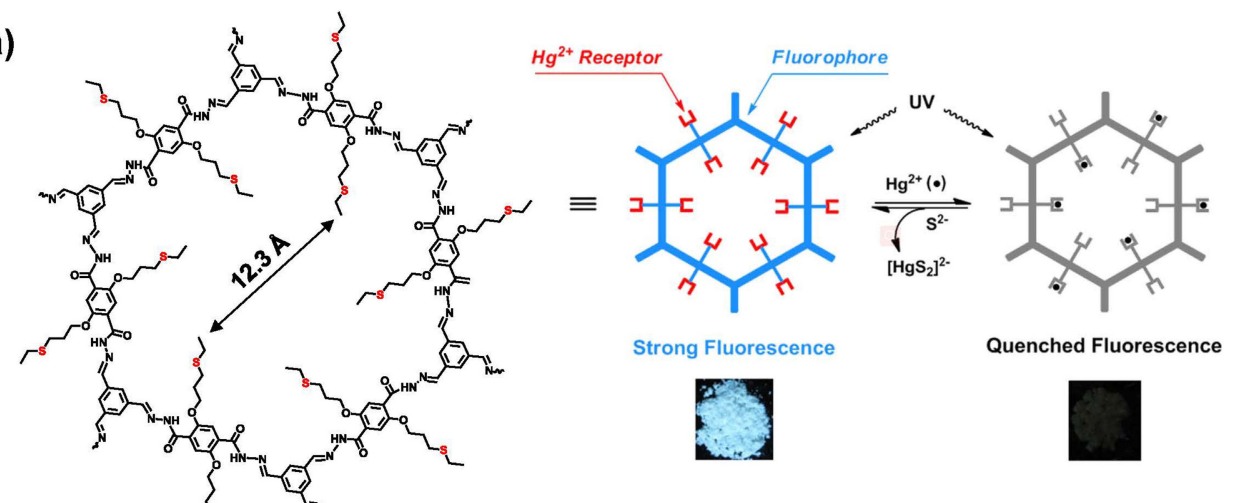

b)

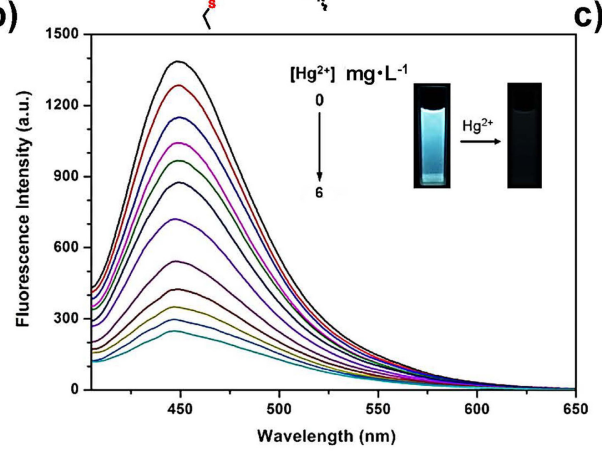

c)

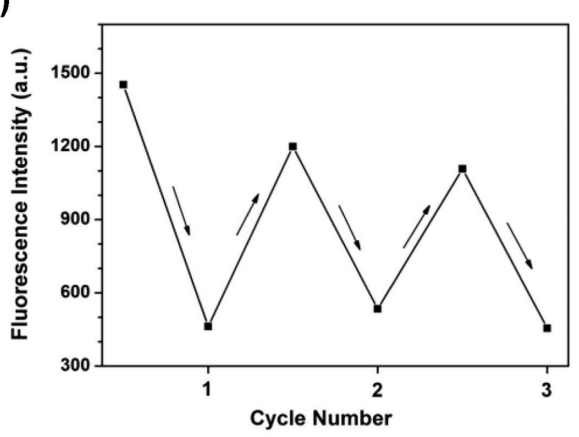

图 4 (a) COF-LZU8 的结构与检测过程示意图、 (b) COF-LZU8 随 $\mathrm{Hg}^{2+}$ 浓度增加的苂光光谱和(c) COF-LZU8 的循环检测

Figure 4 (a) Schematic of the structure of COF-LZU8 and its sensing produce, (b) fluorescence spectrum of COF-LZU8 with increasing $\mathrm{Hg}^{2+}$ concentration, and (c) recycling performance

a)

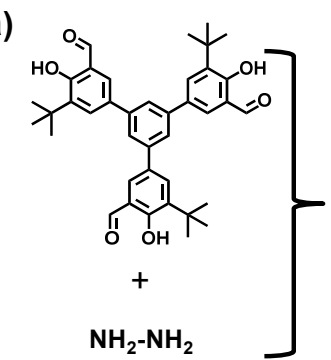

b)

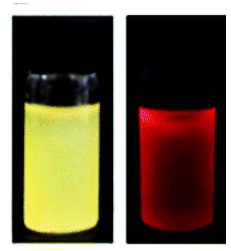

c)

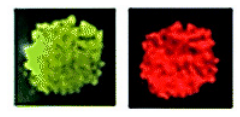

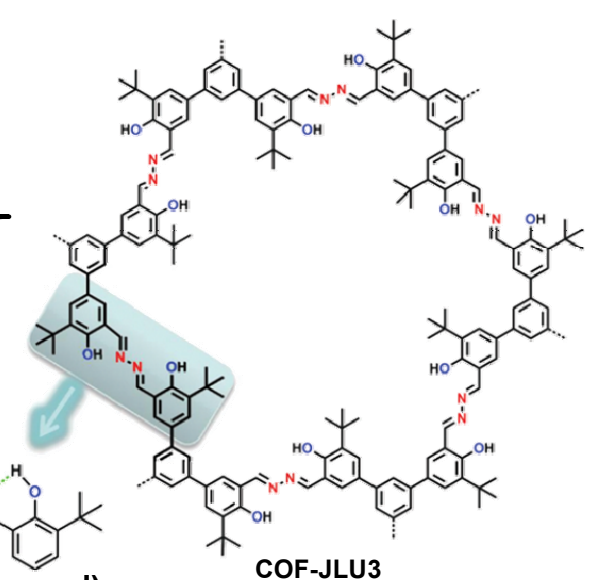

d)

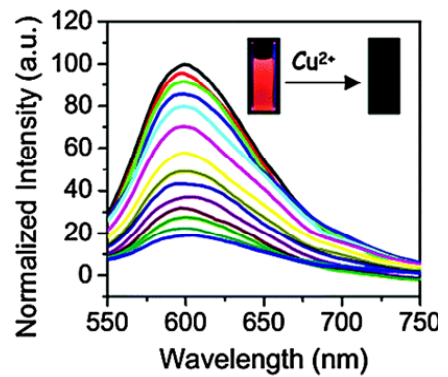

图 5 (a) COF-JLU3 的合成与结构示意图、(b) $360 \mathrm{~nm}$ 紫外光下 COF-JLU3 分散于 PEG 中的图像、(c) $360 \mathrm{~nm}$ 紫外光下 COF-JLU3 固态下的图像和(d) COF-JLU3 在不同 $\mathrm{Cu}^{2+}\left(0 \sim 12 \mathrm{mg} \cdot \mathrm{L}^{-1}\right)$ 浓度下苂光光谱

Figure 5 (a) Synthesis and structure of COF-JLU3, (b) images of COF-JLU3 suspended in PEG under UV-light irradiation of $360 \mathrm{~nm}$, (c) images of COF-JLU3 in solid state under UV-light irradiation of $360 \mathrm{~nm}$, and (d) luminescence spectra of COF-JLU3 in THF suspensions containing different concentrations of $\mathrm{Cu}^{2+}\left(0 \sim 12 \mathrm{mg} \cdot \mathrm{L}^{-1}\right)$ 
$\mathrm{Cu}^{2+}$ 的检出限为 $19 \mathrm{mg} \cdot \mathrm{L}^{-1}$, 且其在 2.0 equiv. $\mathrm{Cu}^{2+}$ 溶液 中, 发光强度降低 $81.7 \%$ (图 5d). 此外, 得益于 COF-JLU3 优异的结晶性、较高的孔隙率、良好的发光 效率及大量的作用位点, COF-JLU3 对 $\mathrm{Cu}^{2+}$ 离子具有很 好的灵敏度与选择性.

除可用于重金属阳离子的检测, COFs 还可用于重 金属氧酸盐阴离子的传感. 最近, 施展等 ${ }^{[20]}$ 设计并合成 了具有苂光性能的 COF-TT. 研究发现, $\mathrm{Fe}^{3+}$ 等阳离子可 通过路易斯酸碱相互作用与 COF-TT 骨架发生电子交 换, 使其苂光猝灭. 而 $\mathrm{CrO}_{4}^{2-}, \mathrm{Cr}_{2} \mathrm{O}_{7}^{2-}$ 和 $\mathrm{MnO}_{4}^{-}$等阴离 子则可吸收 COF-TT 中大部分有机配体的激发能, 从而 使其荧光减弱甚至猝灭, 以此用于 $\mathrm{Fe}^{3+}, \mathrm{CrO}_{4}^{2-}$, $\mathrm{Cr}_{2} \mathrm{O}_{7}^{2-}$ 和 $\mathrm{MnO}_{4}^{-}$等离子的传感. 值得一提的是, COFTT 在 $\mathrm{pH}$ 约为 2 至 14 范围内表现出极高的化学稳定性, 具有潜在的实际应用价值.

\section{$1.4 \mathrm{COFs}$ 在 $\mathrm{pH}$ 传感中的应用}

环境中 $\mathrm{pH}$ 的改变对天气和土壤等均会产生巨大影 响 ${ }^{[21]}$ (如酸雨和土壤酸化). 此外, $\mathrm{pH}$ 也是食品、药品和 饮用水等产品的重要指标 ${ }^{[22]}$. 本节将对 $\mathrm{COFs}$ 在 $\mathrm{pH}$ 传 感中的应用进行总结与讨论 ${ }^{[23]}$.

2016 年, 刘晓明等 ${ }^{[23 a]}$ 利用框架中氮原子的质子化/ 去质子化过程首次报道了可用于 $\mathrm{pH}$ 传感的 COF (COFJLU4). 研究发现, COF-JLU4 的发射波长和强度可在 $\mathrm{pH}$ $0.9 \sim 13.0$ 范围内呈现连续变化, 且其位于 $428 \mathrm{~nm}$ 处的
荧光强度随着环境中 $\mathrm{pH}$ 的降低而逐渐升高. $\mathrm{COF}$ 总体 的荧光强度则在 $\mathrm{pH}$ 为 $9.0 \sim 13.0$ 以及 $0.9 \sim 4.5$ 的区间内 有一定的线性关系，且随着 $\mathrm{pH}$ 的降低而出现明显的升 高. 此外, COF-JLU4 在 $\mathrm{pH}$ 荧光传感过程中还表现出良 好的可重复使用性.

为进一步探索 $\mathrm{COFs}$ 在 $\mathrm{pH}$ 快捷检测中的潜在应用, 王殳等 ${ }^{[23 b]}$ 将酚羟基引入到 $\mathrm{COFs}$ 中合成了 8-差基喹 啉功能化的 COF-HQ(图 6a). 研究发现, COF-HQ 在水和 酸性溶液中均表现出良好的稳定性，可用于各种水体环 境中的 $\mathrm{pH}$ 检测. 此外, 检测结果表明, 当 $\mathrm{pH}=1$ 时, COF-HQ 的荧光完全猝灭; 在 $\mathrm{pH}$ 值为 $1 \sim 5$ 的溶液中, COF-HQ 的荧光强度随着 $\mathrm{pH}$ 的升高而增强, 并显示出 良好的线性关系 $\left(\mathrm{R}^{2}=0.979\right.$, 图 $\left.6 \mathrm{~b}\right)$. 值得注意的是, COF-HQ 在检测过程中展现出肉眼可见的颜色变化(从 黄色变为黑色, 图 6d), 且在五次循环实验中保持骨架 完整，说明该 $\mathrm{COF}$ 可用于 $\mathrm{pH}$ 的肉眼可见快捷检测.

\subsection{COFs 在生物传感中的应用}

生物体内各种生物分子的表达水平对生命活动的 揭示具有重要作用, COFs 在这些生物分子的传感中也 具有潜在应用 ${ }^{[24]}$. 本节将对 COFs 材料在生物传感方面 的应用进行综述.

表皮生长因子受体(EGFR)是一种跨膜蛋白, 其异 常表达通常与肺癌、乳腺癌、前列腺癌等癌症相关 ${ }^{[25]}$. 因此, 开发可用于 EGFR 表达水平原位检测的材料具有 a)

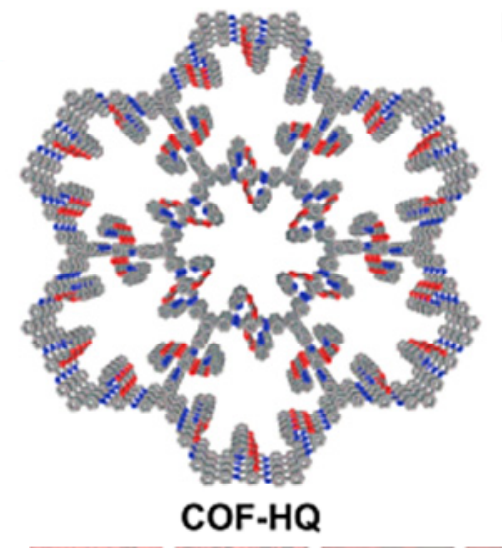

c)

d)
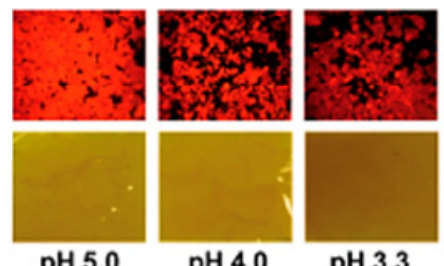

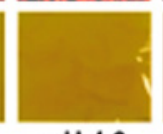

pH 4.0

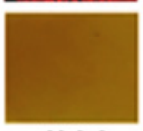

pH 3.3 b)

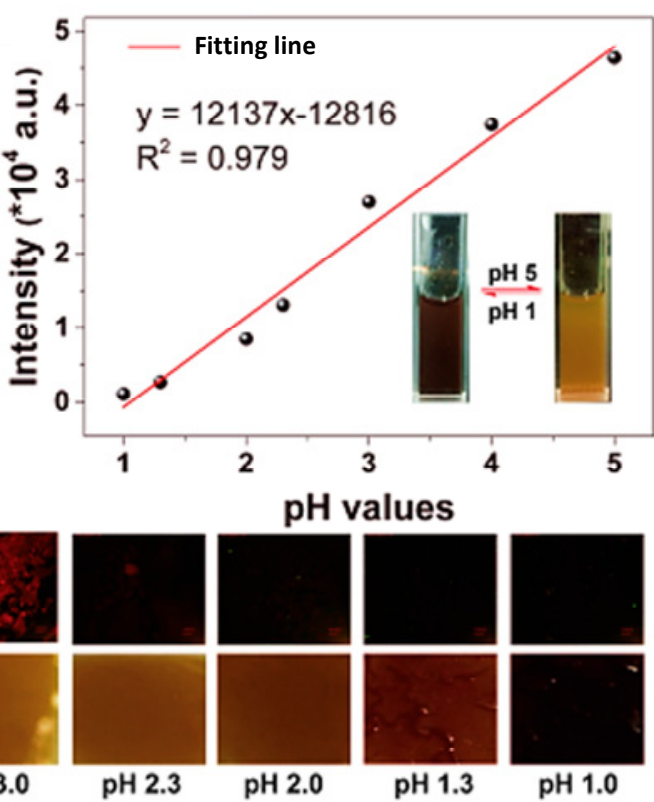

图 6 (a) COF-HQ 结构示意图、(b) COF-HQ 苂光强度与 $\mathrm{pH}$ 值的线性关系、(c) COF-HQ 在不同酸性 $\mathrm{pH}$ 溶液中的苂光图像和(d) COF-HQ 在不同酸性 $\mathrm{pH}$ 溶液中的颜色变化

Figure 6 (a) Schematic diagram of the structure of COF-HQ, (b) the linear relationship between $\mathrm{pH}$ and fluorescence intensity, (c) images of the fluorescence of COF-HQ after treatment with various acidic solutions, and (d) naked eye color changes after treatment with various acidic solutions 
重大意义. 2019 年, 刘仲毅课题组 ${ }^{[26]}$ 报道了具有优异的 电化学活性、良好的生物相容性和低毒性的卟啉基 COF ( $p$-COF), 并以此为基础构筑了可用于 EGFR 和人乳腺 癌细胞 MCF-7 超灵敏检测的电化学适体传感器. 电化 学实验结果表明, 基于 $p$-COF 的适体传感器对 EGFR 的 检测限低至 $5.64 \mathrm{fg} \cdot \mathrm{mL}^{-1}$ (差分脉冲伏安法) 和 7.54 $\mathrm{fg} \cdot \mathrm{mL}^{-1}$ (电化学阻抗法), 对 MCF-7 的检测限为 61 cell $\mathrm{mL}^{-1}$. 此外, $p$-COF 适体传感器同时表现出优异的选择 性、良好的稳定性、可重复性以及在人血清样品中的适 用性. 该研究表明, 以 COFs 材料为基础构筑的生物传 感器可为目标癌症标志物的检测提供新的思路.

硫化氢 $\left(\mathrm{H}_{2} \mathrm{~S}\right)$ 作为生命体内一种重要的信号分子 ${ }^{[27]}$, 在神经退行性疾病、衰老和肿瘤扩散等生理过程中发挥 着至关重要的作用. 2018 年, 张晓兵等 ${ }^{[27]}$ 利用后合成修 饰策略将双光子苂光探针 NPHS 引入到 COF 中, 构筑了 具有低细胞毒性、出色的光稳定性和长期生物成像能力 的 TpASH-NPHS, 并通过超声剥离的方法进一步构筑 了纳米尺度的生物传感器. 与另外一种已报道的 $\mathrm{H}_{2} \mathrm{~S}$ 小 分子荧光探针 CNPHS 相比, TpASH-NPHS 不仅在体外 试验中表现出更好的稳定性及相似的传感性能, 还可通 过其孔道的尺寸 $(1.3 \mathrm{~nm})$ 限制细胞内酶的进入从而排除 其对 $\mathrm{H}_{2} \mathrm{~S}$ 检测的干扰(图 7a). 此外, TpASH-NPHS 作为
一种新型双光子荧光探针，可在近红外光谱(NIR)的激 发下实现活体细胞以及深层组织中 $\mathrm{H}_{2} \mathrm{~S}$ 的检测和成像, 在大大减少细胞自体荧光的影响和对组织伤害的同时 增加了组织穿透深度 (图 7b, 7c). 此研究表明, 荧光 COFs 纳米探针在生物传感和纳米医学等研究领域具有 重大的应用前景.

核酸检测 ${ }^{[28]}$ 在基因工程和生物医学领域具有重要 作用. 2017 年, Zhang 等 ${ }^{[29]}$ 通过柔性单元的引入构筑了 层间堆叠作用较弱的 TPA-COF，并通过简单的方式高 产率制备了超薄二维纳米薄片(TPA-COF NSs). 随后, Zhang 等设计了 $\mathrm{H}_{1}$ (染料标记)和 $\mathrm{H}_{2}$ 两种发夹型 DNA 探 针, 通过 $\pi-\pi$ 相互作用, $\mathrm{H}_{1}$ 和 $\mathrm{H}_{2}$ 可吸附在 TPA-COF NSs 表面，使染料苂光猝灭，从而作为 DNA 的苂光检测探 针. 当存在目标 DNA 时, 探针产生与 TPA-COF NSs 相 互作用较弱的双链 DNA, 从 TPA-COF NSs 表面离去, 使染料苂光恢复, 从而实现目标 DNA 的检测. 检测结 果表明, 基于 TPA-COF NSs 所构筑探针的苂光强度与 目标 DNA 在 $0 \sim 1 \mathrm{nmol} / \mathrm{L}$ 浓度范围内呈良好的线性正 相关, 检出限为 $20 \mathrm{pmol} / \mathrm{L}$, 优于或与大多数基于二维 纳米材料的荧光 DNA 传感器相当, 说明 COFs 在核酸检 测中也具有较好的应用前景. 类似地, 严秀平等 ${ }^{[30]}$ 也将 COF 用于 DNA 的苂光增强检测.
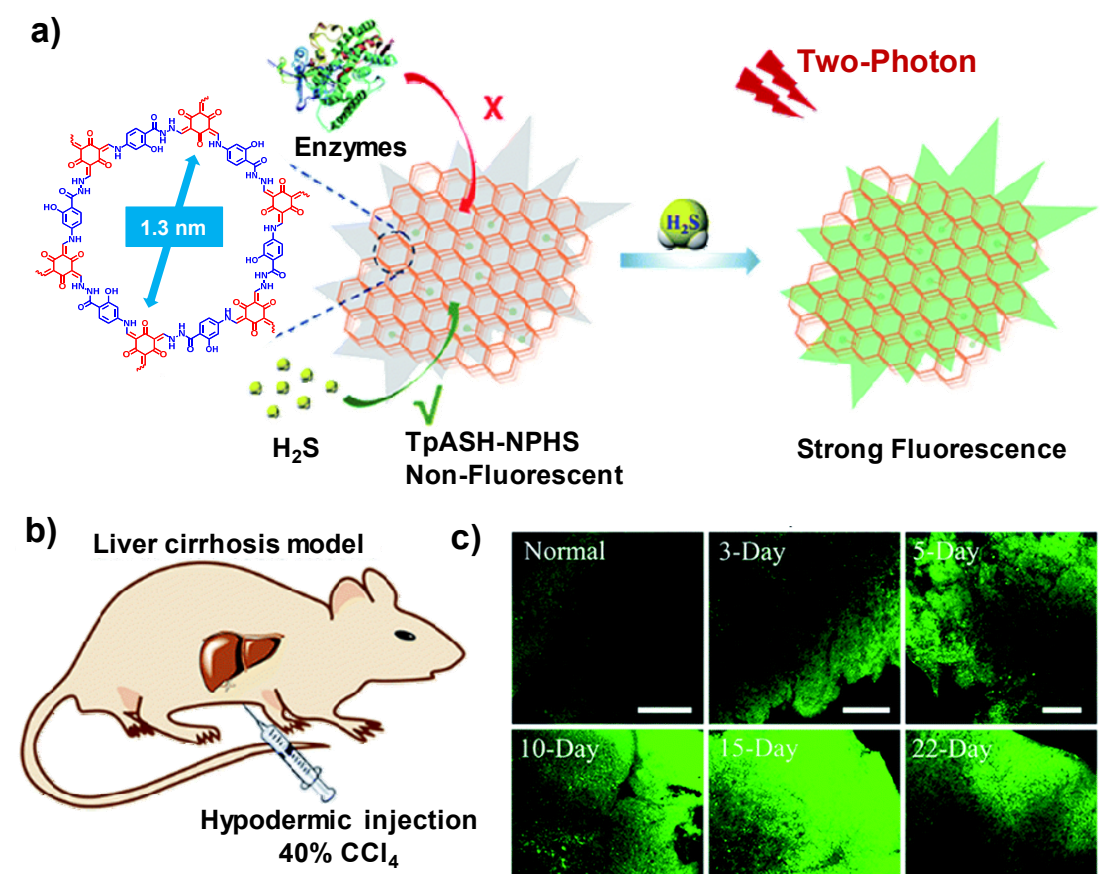

c) Normal

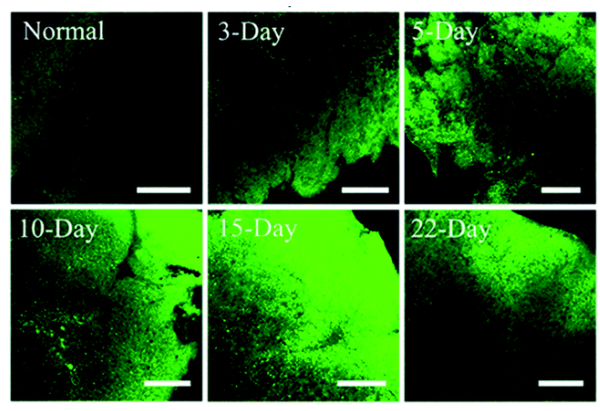

图 7 (a) TpASH-NPHS 的组成和作用方式、(b)皮下注射 40\% $\mathrm{CCl}_{4}$ 诱导肝硬化小鼠模型和(c)不同处理时期肝硬化小鼠肝组织中 TpASH-NPHS 的双光子共聚焦苂光图像(比例尺: $300 \mu \mathrm{m}$ )

Figure 7 (a) Construction of COF-based nanoprobe TpASH-NPHS, (b) the liver cirrhosis mouse model induced by subcutaneous injection of $40 \% \mathrm{CCl}_{4}$, and (c) two-photon confocal fluorescence images of TpASH-NPHS in liver tissues of the cirrhotic mouse model at different $\mathrm{CCl}_{4}$-treated periods (Scale bar: $300 \mu \mathrm{m}$ ) 


\section{$1.6 \mathrm{COFs}$ 在气体传感中的应用}

挥发性气体如富电子芳烃类分子及酸碱类腐蚀性 气体 $\left(\mathrm{HCl}, \mathrm{NH}_{3}\right)$ 等都极易在空气中扩散, 危害生态环境 和人类健康 ${ }^{[31]}$. COFs 作为一种可设计性强的有机多孔 材料, 有利于气体分子进入孔道中, 进而与 $\mathrm{COFs}$ 骨架 中的功能基团发生作用, 实现气体传感过程 ${ }^{[32]}$. 本部分 将对 COFs 在气体传感方面的应用进行综述.

根据主客体相互作用, COFs 本身性质在气体分子 进入孔道中与功能位点作用后发生变化, 利用该性质的 变化即可实现气体分子的传感. 2015 年, 郑企雨等 ${ }^{[32 a]}$ 利 用三嗪衍生物构筑了苂光 TAT-COF-2. 研究发现, TATCOF-2 对富电子芳烃气体分子和缺电子芳烃气体分子 分别显示出快速的荧光增强和猝灭行为. 室温环境下, TAT-COF-2 薄层样品置于饱和富电子芳烃蒸气中时, 富 电子芳烃气体分子的电子云可能流向 TAT-COF-2, 使其 苂光明显增强; 而当 TAT-COF-2 薄层样品置于饱和缺电 子芳烃蒸气中时, TAT-COF-2 电子云可能流向缺电子芳 烃气体分子, TAT-COF-2 苂光减弱, 从而实现芳烃气体 分子的传感

此外, COFs 还可用于挥发性酸碱气体的检测. 2019 年, Bojdys 等 ${ }^{[22 b]}$ 通过三嗪环为核心的前体构筑了 $\beta$-氨 基烯酮连接的 PBHP-TAPT COF. 研究发现, $\mathrm{HCl}$ 气体分
子进入 PBHP-TAPT COF 的孔道中可使骨架中的三嗪功 能单元质子化, 进而使 PBHP-TAPT COF $\pi$ 电子云密度 发生改变, 颜色由橙色加深至红色, 电导率提高两个数 量级. 而将 $\mathrm{HCl}$ 蒸气接触后的 PBHP-TAPT COF 样品置 于 $\mathrm{NH}_{3}$ 蒸气中时, PBHP-TAPT COF 的性质又可恢复(图 $8 \mathrm{a} \sim 8 \mathrm{c})$, 且该过程在 PBHP-TAPT COF 结构完整的条件 下可重复进行至少 5 次, 表明 COFs 可潜在用于挥发性 酸碱气体的实时传感. 最近, 赵新等 ${ }^{[33]}$ 利用四苯乙烯为 核心的前体和对苯二胺，也成功构筑了对 $\mathrm{HCl} / \mathrm{NH}_{3}$ 具有 响应性的 COF, 并将其用于酸碱气体分子的检测. 类似 地，郝策等 ${ }^{[34]}$ 也探究了 $\mathrm{Ph}-\mathrm{An}-\mathrm{COF}$ 对 $\mathrm{NH}_{3}$ 蒸气传感原 理的理论模拟

除对挥发性芳香分子和酸碱气体分子的检测，通过 功能化定向设计, COFs 还可用于特定气体分子的传感. 2019 年，章伟光等 ${ }^{[35]}$ 合成了具有羟基的 BABE-TFPy $\mathrm{COF}$, 并将其涂覆到石英晶体微天平 (QCM)表面，构筑 了对芥子气(2-chloroethyl ethyl sulfide, CEES)具有传感 能力的传感器. 为探究该 QCM 传感器的检测范围, 章 伟光等选定 $40{ }^{\circ} \mathrm{C}$ 为试验条件, 探究了不同涂层量下响 应程度与 CEES 浓度之间的关系. 实验结果表明, 所构 筑传感器可对 $5.6 \sim 39.5 \mathrm{mg} / \mathrm{L}$ 浓度范围内的 CEES 选择 性地快速检测. 且当 CEES 浓度为 $5.6 \sim 19.7 \mathrm{mg} / \mathrm{L}$ 时,

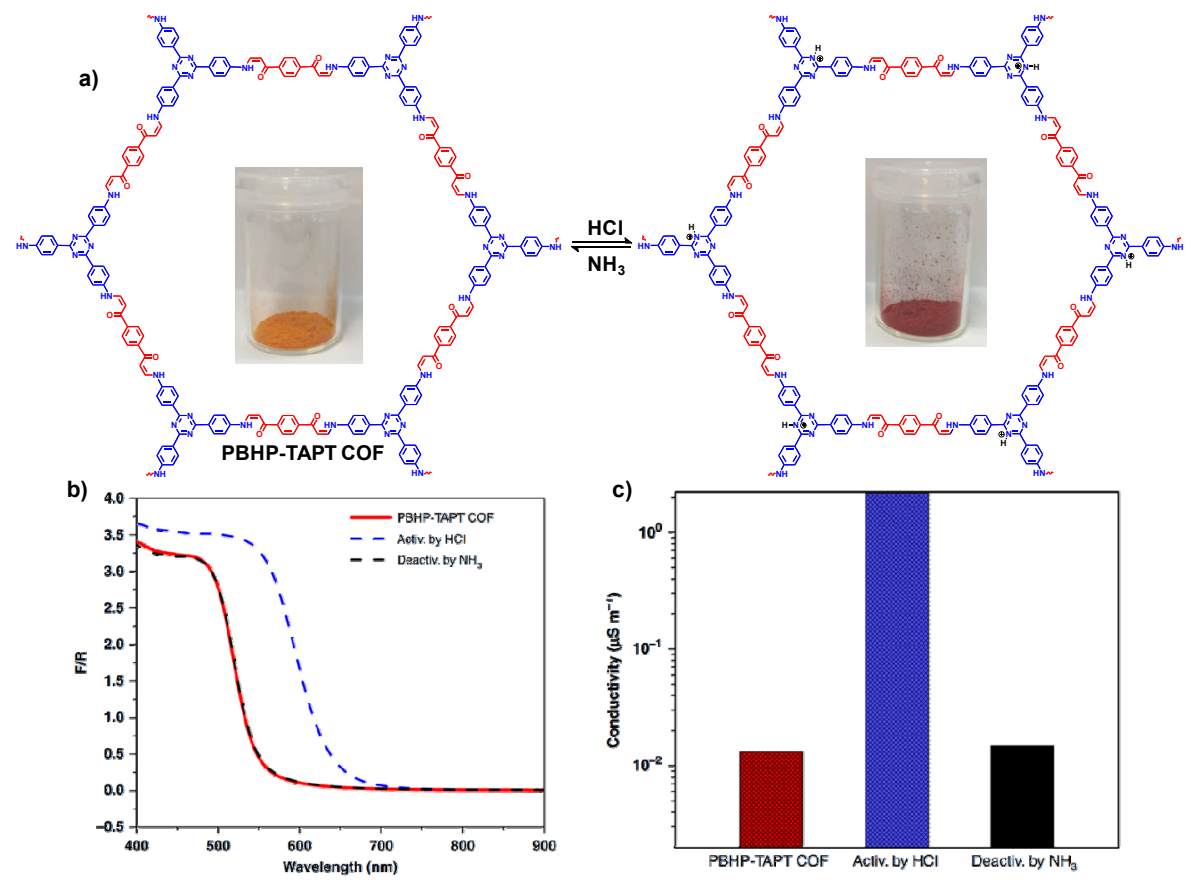

图 8 (a) PBHP-TAPT COF 对酸碱气体的传感机理及可见光下颜色变化, (b) PBHP-TAPT COF 接触酸、碱气体后的固体紫外可见 光谱, (c) PBHP-TAPT COF 接触酸、碱气体后的电导率

Figure 8 (a) Protonation and deprotonation of the triazines with chemical triggers $\left(\mathrm{HCl}\right.$ and $\mathrm{NH}_{3}$ vapours); (b) solid-state UV/Vis spectra of pristine PBHP-TAPT COF (red), after activation with $\mathrm{HCl}$ vapours (blue; dashed and dotted line), and regeneration with $\mathrm{NH}_{3}$ vapours (black; dashed line); (c) electrical conductivity of pristine PBHP-TAPT COF (red), after activation with $\mathrm{HCl}$ vapours (blue), and regeneration with $\mathrm{NH}_{3}$ vapours (black) 
该传感器的传感信号与 CEES 蒸气浓度之间表现出良好 的线性关系 [相关系数 $\left(R^{2}\right)$ 为 0.993 ], 检测限可达 0.96 $\mathrm{mg} / \mathrm{L}$. 此外, 该传感器不仅对 CEES 表现出较高的选择 性, 还可至少循环使用 3 次. 章伟光等认为 CEES 所存 在的硫醚基团以及氯原子可与 BABE-TFPy COF 骨架形 成特殊的双重氢键, 从而使该传感器对 CEES 展现出优 异选择性.

\section{2 总结与展望}

共价有机框架 (Covalent Organic Frameworks, COFs)作为一种新型的有机多孔材料，因其规整开放的 孔道结构、优异的稳定性、较高的比表面积以及较强的 可设计性，已在传感领域的应用中取得了一系列进展 (图 9).
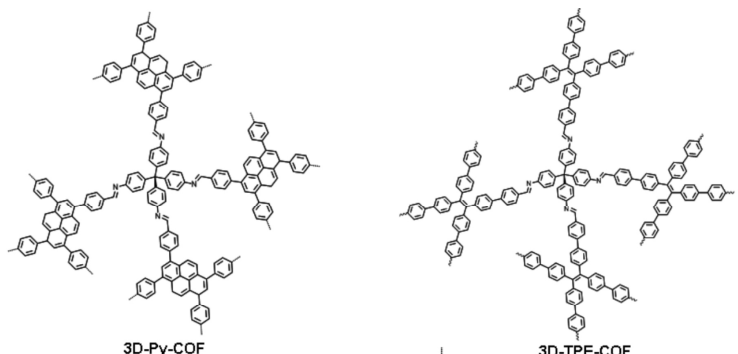

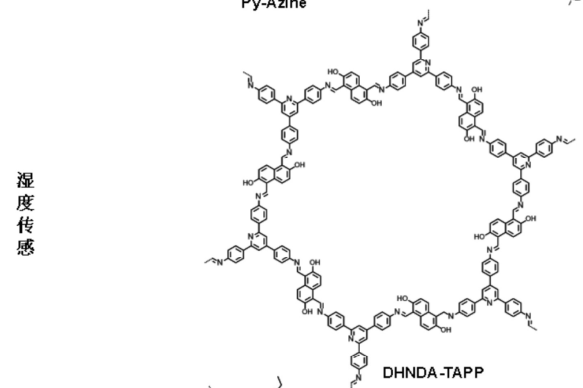

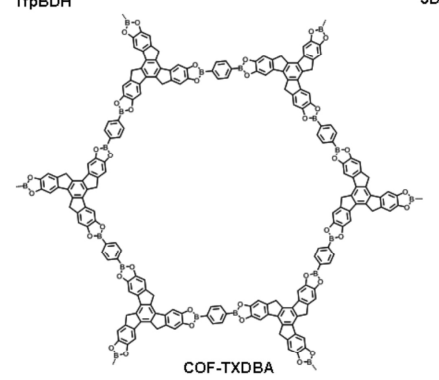

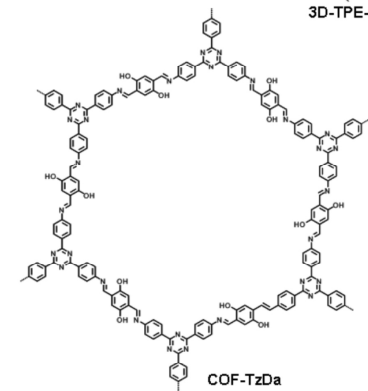

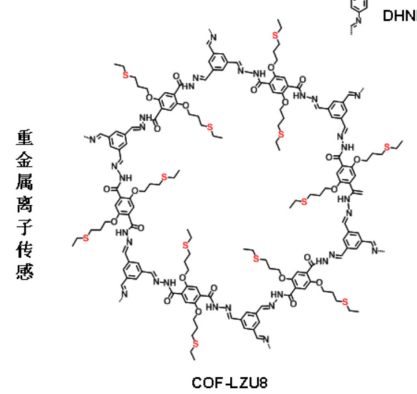

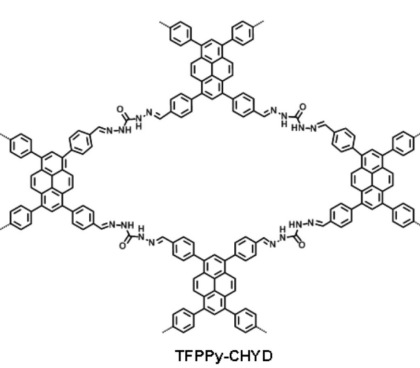

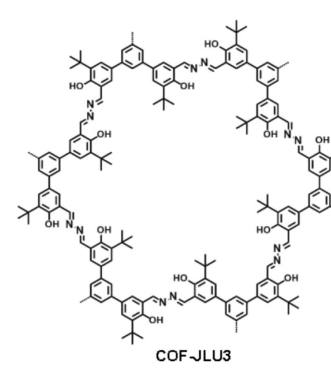

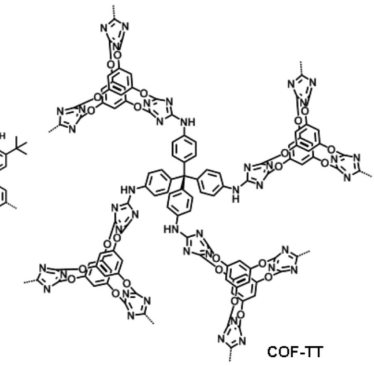

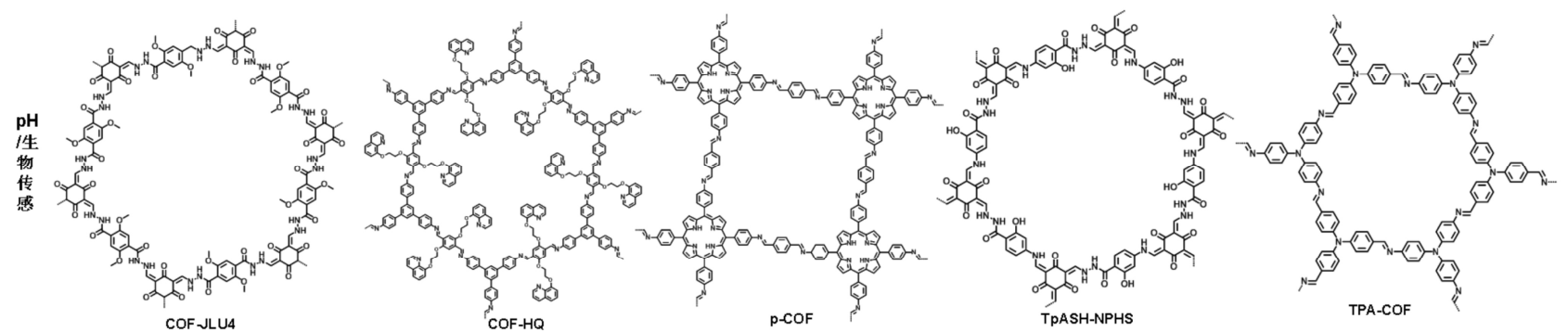

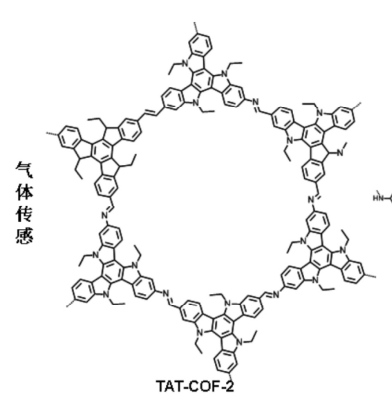

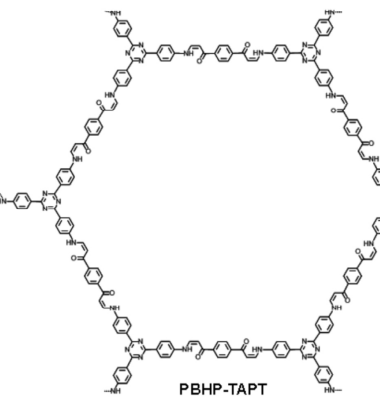

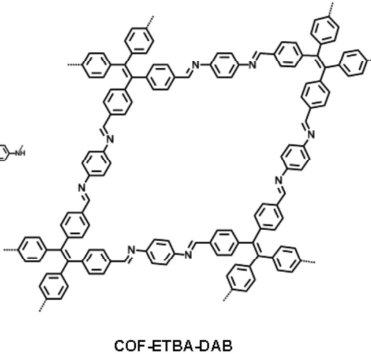

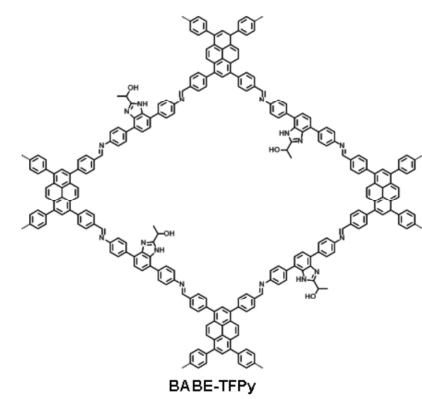

图 9 本文所涉及 $\mathrm{COFs}$ 的结构简图

Figure 9 Simplified structures of these COFs for sensing 
本文主要综述了 COFs 在爆炸物传感、湿度传感、 金属离子传感、 $\mathrm{pH}$ 传感、生物传感、气体传感等领域 中的研究进展. 尽管 COFs 在传感领域的应用中展现出 了较好的设计性、较高的稳定性及循环检测性等独特优 势，但不可否认 COFs 在传感领域的应用还存在以下挑 战: (1)怎样构筑具有较高苂光量子产率的 COFs? 目前 所报道的荧光 COFs 数量较少, COFs 的荧光量子产率普 遍较低. (2)如何进一步增加 COFs 的传感性能? COFs 的 传感性能与小分子相比, 仍有待进一步提高. (3)能否进 一步提高 COFs 与其他材料的复合性? 将 COFs 剥离成 薄片或将其制备成薄膜有利于其更好的同其他材料复 合，有利于克服 COFs 本身的缺陷并提升 COFs 在传感 中的应用.

总之, COFs 作为一种具有重大潜力且快速发展的 新型晶态有机多孔材料, 其在传感领域表现出了广阔的 应用前景. 相信随着更多化学、物理和材料学家的加入, COFs 领域将取得重大进展，同时将有更多灵敏度高、 选择性好和循环稳定性强的 COFs 传感器出现, 不断推 动传感领域的发展.

\section{References}

[1] (a) Ding, S. Y.; Wang, W. Chem. Soc. Rev. 2013, 42, 548

(b) Diercks, C. S.; Yaghi, O. M. Science 2017, 355, 923.

(c) Chen, X.; Geng, K.; Liu, R.; Tan, K. T.; Gong, Y.; Li, Z.; Tao, S.; Jiang, Q.; Jiang, D. Angew. Chem. Int. Ed. 2020, 59, 5050.

[2] Cooper, A. I. Adv. Mater. 2009, 21, 1291.

[3] Yuan, Y.; Zhu, G. ACS Cent. Sci. 2019, 5, 409.

[4] Tan, L.; Tan, B. Chem. Soc. Rev. 2017, 46, 3322.

[5] Baldwin, L. A.; Crowe, J. W.; Pyles, D. A.; McGrier, P. L. J. Am. Chem. Soc. 2016, 138, 15134.

[6] Côté, A. P.; Benin, A. I.; Ockwig, N. W.; O'Keeffe, M.; Matzger, A. J.; Yaghi, O. M. Science 2005, 310, 1166.

[7] (a) Li, Y.; Ben, T.; Qiu, S. Acta Chim. Sinica 2015, 73, 605 (in Chinese).

(李艳强，贲腾，表式纶，化学学报, 2015, 73, 605.)

(b) Zeng, Y.; Zou, R.; Zhao, Y. Adv. Mater. 2016, 28, 2855.

(c) Yuan, S.; Li, X.; Zhu, J.; Zhang, G.; Van Puyvelde, P.; Van der Bruggen, B. Chem. Soc. Rev. 2019, 48, 2665.

(d) Gao, C.; Li, J.; Yin, S.; Lin, G.; Ma, T.; Meng, Y.; Sun, J.; Wang, C. Angew. Chem., Int. Ed. 2019, 58, 9770.

[8] (a) Xu, H. S.; Ding, S. Y.; An, W. K.; Wu, H.; Wang, W. J. Am. Chem. Soc. 2016, 138, 11489.

(b) Han, X.; Xia, Q.; Huang, J.; Liu, Y.; Tan, C.; Cui, Y. J. Am. Chem. Soc. 2017, 139, 8693.

(c) Wei, P. F.; Qi, M. Z.; Wang, Z. P.; Ding, S. Y.; Yu, W.; Liu, Q.; Wang, L. K.; Wang, H. Z.; An, W. K.; Wang, W. J. Am. Chem. Soc. 2018, 140, 4623.

(d) Chen, R.; Shi, J. L.; Ma, Y.; Lin, G.; Lang, X.; Wang, C. Angew. Chem., Int. Ed. 2019, 58, 6430.

(e) Sharma, R. K.; Yadav, P.; Yadav, M.; Gupta, R.; Rana, P.; Srivastava, A.; Zbořil, R.; Varma, R. S.; Antonietti, M.; Gawande, M. B. Mater. Horiz. 2020, 7, 411 .

[9] (a) Bertrand, G. H.; Michaelis, V. K.; Ong, T. C.; Griffin, R. G.; Dinca, M. Proc. Natl. Acad. Sci. U. S. A. 2013, 110, 4923.

(b) Dogru, M.; Handloser, M.; Auras, F.; Kunz, T.; Medina, D.; Hartschuh, A.; Knochel, P.; Bein, T. Angew. Chem., Int. Ed. 2013, 52,2920 .

(c) Bessinger, D.; Ascherl, L.; Auras, F.; Bein, T. J. Am. Chem. Soc.
2017, 139, 12035 .

(d) Jin, E.; Asada, M.; Xu, Q.; Dalapati, S.; Addicoat, M. A.; Brady, M. A.; Xu, H.; Nakamura, T.; Heine, T.; Chen, Q.; Jiang, D. Science 2017, 357, 673 .

(e) Ding, H.; Li, J.; Xie, G.; Lin, G.; Chen, R.; Peng, Z.; Yang, C.; Wang, B.; Sun, J.; Wang, C. Nat. Commun. 2018, 9, 5234.

[10] (a) Das, G.; Biswal, B. P.; Kandambeth, S.; Venkatesh, V.; Kaur, G.; Addicoat, M.; Heine, T.; Verma, S.; Banerjee, R. Chem. Sci. 2015 , 6, 3931

(b) Ding, S. Y.; Dong, M.; Wang, Y. W.; Chen, Y. T.; Wang, H. Z.; Su, C. Y.; Wang, W. J. Am. Chem. Soc. 2016, 138, 3031.

(c) Li, Z.; Huang, N.; Lee, K. H.; Feng, Y.; Tao, S.; Jiang, Q.; Nagao, Y.; Irle, S.; Jiang, D. J. Am. Chem. Soc. 2018, 140, 12374.

(d) Liu, X.; Huang, D.; Lai, C.; Zeng, G.; Qin, L.; Wang, H.; Yi, H.; Li, B.; Liu, S.; Zhang, M.; Deng, R.; Fu, Y.; Li, L.; Xue, W.; Chen, S. Chem. Soc. Rev. 2019, 48, 5266.

[11] (a) Liao, H.; Ding, H.; Li, B.; Ai, X.; Wang, C. J. Mater. Chem. A 2014, 2,8854 .

(b) Wang, S.; Wang, Q.; Shao, P.; Han, Y.; Gao, X.; Ma, L.; Yuan, S.; Ma, X.; Zhou, J.; Feng, X.; Wang, B. J. Am. Chem. Soc. 2017, $139,4258$.

(c) Mulzer, C. R.; Shen, L.; Bisbey, R. P.; McKone, J. R.; Zhang, N.; Abruna, H. D.; Dichtel, W. R. ACS Cent. Sci. 2016, $2,667$.

(d) Zhou, J.; Wang, B. Chem. Soc. Rev. 2017, 46, 6927.

(e) Peng, Z.; Ding, H.; Chen, R.; Gao, C.; Wang, C. Acta Chim. Sinica 2019, 77, 681 (in Chinese).

(彭正康, 丁慧敏，陈如凡，高超，汪成，化学学报，2019，77, 681.)

[12] (a) Kandambeth, S.; Dey, K.; Banerjee, R. J. Am. Chem. Soc. 2019 , 141, 1807.

(b) Chen, X.; Geng, K.; Liu, R.; Tan, K. T.; Gong, Y.; Li, Z.; Tao, S.; Jiang, Q.; Jiang, D. Angew. Chem., Int. Ed. 2019, 58, 2.

(c) Zhou, B.; Chen, L. Acta Chim. Sinica 2015, 73, 487 (in Chinese).

(周宝龙, 陈龙, 化学学报, 2015, 73, 487.)

[13] (a) Wan, S.; Guo, J.; Kim, J.; Ihee, H.; Jiang, D. Angew. Chem., Int. Ed. 2008, 47, 8826 .

(b) Dalapati, S.; Gu, C.; Jiang, D. Small 2016, 12, 6513.

(c) Dong, J.; Li, X.; Peh, S. B.; Yuan, Y. D.; Wang, Y.; Ji, D.; Peng, S.; Liu, G.; Ying, S.; Yuan, D.; Jiang, J.; Ramakrishna, S.; Zhao, D. Chem. Mater. 2018, 31, 146.

(d) Dalapati, S.; Jin, E.; Addicoat, M.; Heine, T.; Jiang, D. J. Am Chem. Soc. 2016, 138, 5797.

[14] (a) Zhang, W.; Qiu, L. G.; Yuan, Y. P.; Xie, A. J.; Shen, Y. H.; Zhu, J. F. J. Hazard. Mater. 2012, 221, 147.

(b) Gao, Q.; Li, X.; Ning, G. H.; Leng, K.; Tian, B.; Liu, C.; Tang, W.; Xu, H. S.; Loh, K. P. Chem. Commun. 2018, 54, 2349.

(c) Kaleeswaran, D.; Vishnoi, P.; Murugavel, R. J. Mater. Chem. C 2015, 3, 7159 .

(d) Dalapati, S.; Jin, S.; Gao, J.; Xu, Y.; Nagai, A.; Jiang, D. J. Am. Chem. Soc. 2013, 135, 17310.

(e) Lin, G.; Ding, H.; Yuan, D.; Wang, B.; Wang, C. J. Am. Chem. Soc. 2016, 138, 3302.

(f) Pang, C.; Luo, S.; Hao, Z.; Gao, J.; Huang, Z.; Yu, J.; Yu, S.; Wang, Z. Chin. J. Org. Chem. 2018, 38, 2606 (in Chinese). (庞楚明，罗时荷，郝志峰，高健，黄召昊，余家海，余思敏，汪 朝阳，有机化学, 2018, 38, 2606.)

[15] (a) Sikarwar, S.; Yadav, B. C. Sens. Actuator, A 2015, 233, 54. (b) Yeo, T. L.; Sun, T.; Grattan, K. T. V. Sens. Actuator, A 2008 $144,280$.

[16] (a) Jhulki, S.; Evans, A. M.; Hao, X. L.; Cooper, M. W.; Feriante, C. H.; Leisen, J.; Li, H.; Lam, D.; Hersam, M. C.; Barlow, S.; Bredas, J. L.; Dichtel, W. R.; Marder, S. R. J. Am. Chem. Soc. 2020 , 142, 783 .

(b) Ascherl, L.; Evans, E. W.; Hennemann, M.; Di Nuzzo, D.; Hufnagel, A. G.; Beetz, M.; Friend, R. H.; Clark, T.; Bein, T.; Auras, F. Nat. Commun. 2018, 9, 3802.

(c) Huang, W.; Jiang, Y.; Li, X.; Li, X.; Wang, J.; Wu, Q.; Liu, X. 
ACS Appl. Mater. Interfaces 2013, 5, 8845.

(d) Singh, H.; Tomer, V. K.; Jena, N.; Bala, I.; Sharma, N.; Nepak, D.; De Sarkar, A.; Kailasam, K.; Pal, S. K. J. Mater. Chem. A 2017, 5, 21820 .

(e) Qian, H. L.; Dai, C.; Yang, C. X.; Yan, X. P. ACS Appl. Mater. Interfaces 2017, 9, 24999.

[17] (a) Bao, B.; Ma, M.; Fan, Q.; Wang, L.; Huang, W. Acta Chim. Sinica 2013, 71, 1379 (in Chinese).

(鲍碧清, 马明风, 范曲立, 汪联辉, 黄维, 化学学报, 2013, 71, 1379.)

(b) Hu, C.; Huang, D.; Zeng, G.; Cheng, M.; Gong, X.; Wang, R.; Xue, W.; Hu, Z.; Liu, Y. Chem. Eng. J. 2018, 338, 432.

[18] (a) Cui, W. R.; Jiang, W.; Zhang, C. R.; Liang, R. P.; Liu, J; Qiu, J. D. ACS Sustainable Chem. Eng. 2020, 8, 445.

(b) Chen, G.; Lan, H. H.; Cai, S. L.; Sun, B.; Li, X. L.; He, Z. H.; Zheng, S. R.; Fan, J.; Liu, Y.; Zhang, W. G. ACS Appl. Mater. Interfaces 2019, 11, 12830.

(c) Wang, T.; Xue, R.; Chen, H.; Shi, P.; Lei, X.; Wei, Y.; Guo, H.; Yang, W. New J. Chem. 2017, 41, 14272.

(d) Zhou, Z.; Zhong, W.; Cui, K.; Zhuang, Z.; Li, L.; Li, L.; Bi, J.; Yu, Y. Chem. Commun. 2018, 54, 9977.

(e) Zhang, T.; Gao, C.; Huang, W.; Chen, Y.; Wang, Y.; Wang, J. Talanta 2018, 188, 578 .

(f) Wang, R.; Ji, W.; Huang, L.; Guo, L.; Wang, X. Anal. Lett. 2019, $52,1757$.

[19] Li, Z.; Zhang, Y.; Xia, H.; Mu, Y.; Liu, X. Chem. Commun. 2016, $52,6613$.

[20] Li, M.; Cui, Z.; Pang, S.; Meng, L.; Ma, D.; Li, Y.; Shi, Z.; Feng, S. J. Mater. Chem. C 2019, 7, 11919.

[21] Zafar, S.; D’Emic, C.; Afzali, A.; Fletcher, B.; Zhu, Y.; Ning, T. Nanotechnology 2011, 22, 405501.

[22] Liu, Y. Y.; Wu, M.; Zhu, L. N.; Feng, X. Z.; Kong, D. M. Chem. Asian J. 2015, 10, 1304.

[23] (a) Zhang, Y.; Shen, X.; Feng, X.; Xia, H.; Mu, Y.; Liu, X. Chem. Commun. 2016, 52, 11088.

(b) Chen, L.; He, L.; Ma, F.; Liu, W.; Wang, Y.; Silver, M. A.; Chen, L.; Zhu, L.; Gui, D.; Diwu, J.; Chai, Z.; Wang, S. ACS Appl. Mater. Interfaces 2018, 10, 15364.

(c) Xu, M.; Wang, L.; Xie, Y.; Song, Y.; Wang, L. Sens. Actuator, B 2019, 281, 1009.

(d) Ma, Y.; Yuan, F.; Yu, Y.; Zhou, Y..; Zhang, X. Anal. Chem. 2020, 92, 1424 .
[24] (a) Zhang, X.; Chi, K. N.; Li, D. L.; Deng, Y.; Ma, Y. C.; Xu, Q. Q.; Hu, R.; Yang, Y. H. Biosens. Bioelectron. 2019, 129, 64.

(b) Wang, J.; Yan, B. Anal. Chem. 2019, 91, 13183.

(c) Li, W.; Yang, C. X.; Yan, X. P. Chem. Commun. 2017, 53, 11469 .

(d) Huang, C.; Chen, H.; Li, F.; An, S. Chin. J. Org. Chem. 2019, 39, 2467 (in Chinese).

(黄池宝, 陈会, 李福琴, 安思雅, 有机化学, 2019, 39, 2467.)

[25] (a) Yang, T.; Cui, Y.; Chen, H.; Li, W. Acta Chim. Sinica 2017, 75, 339 (in Chinese)

(杨涛，崔亚男，陈怀银，李伟华，化学学报, 2017, 75, 339.)

(b) Xiong, M.; Rong, Q.; Meng, H. M.; Zhang, X. B. Biosens. Bioelectron. 2017, 89, 212.

[26] Yan, X.; Song, Y.; Liu, J.; Zhou, N.; Zhang, C.; He, L.; Zhang, Z.; Liu, Z. Biosens. Bioelectron. 2019, 126, 734.

[27] Wang, P.; Zhou, F.; Zhang, C.; Yin, S. Y.; Teng, L.; Chen, L.; Hu, X. X.; Liu, H. W.; Yin, X.; Zhang, X. B. Chem. Sci. 2018, 9, 8402.

[28] Zanoli, L. M.; D'Agata, R.; Spoto, G. Anal. Bioanal. Chem. 2012, 402, 1759.

[29] Peng, Y.; Huang, Y.; Zhu, Y.; Chen, B.; Wang, L.; Lai, Z.; Zhang, Z.; Zhao, M.; Tan, C.; Yang, N.; Shao, F.; Han, Y.; Zhang, H. J. Am. Chem. Soc. 2017, 139, 8698.

[30] Li, W.; Yang, C.; Yan, X. Chem. Commun. 2017, 53, 11469.

[31] Yang, Y.; Zhao, Z.; Yang, Y.; Li, G.; Hao, C. New J. Chem. 2019, 43, 9274.

[32] (a) Xie,Y.; Ding, S.; Liu, J.; Wang, W.; Zheng, Q. J. Mater. Chem. C 2015, 3, 10066.

(b) Kulkarni, R.; Noda, Y.; Kumar Barange, D.; Kochergin, Y. S.; Lyu, P.; Balcarova, B.; Nachtigall, P.; Bojdys, M. J. Nat. Commun. 2019, 10, 3228.

(c) Meng, Z.; Stolz, R. M.; Mirica, K. A. J. Am. Chem. Soc. 2019, 141, 11929.

(d) Pang, Y.; Yue, Q.; Huang, Y.; Yang, C.; Shen, X. Talanta 2020, 206, 120194

(e) Zhang, S.; Yang, Q.; Li, Z. Anal. Bioanal. Chem. 2017, 409, 3429.

[33] Cui, F.; Xie, J.; Jiang, S.; Gan, S.; Ma, D.; Liang, R.; Jiang, G.; Zhao, X. Chem. Commun. 2019, 55, 4550.

[34] Yang, Y.; Zhao, Z.; Yang, Y.; Li, G.; Hao, C. New J. Chem. 2019, 43, 9274.

[35] He, Z.; Gong, S.; Cai, S.; Yan, Y.; Chen, G.; Li, X.; Zheng, S.; Fan, J.; Zhang, W. Cryst. Growth Des. 2019, 19, 3543. 